\title{
Comparing Prostate Cancer Treatment Decision Making in a Resource-Rich and a Resource-Poor Environment: A Tale of Two Hospitals
}




\begin{abstract}
Objective

Black men with prostate cancer are diagnosed later, have poorer treatment outcomes, and higher mortality from the disease than all other racial groups. While existing literature has explored differences in the treatment decision making process between black and white men with localized prostate cancer, little is known about how environmental factors may affect the treatment decision process for men with clinically significant disease for whom treatment improves survival. The aim of this study was to compare and contrast the treatment decision process, from both patients' and treating physicians' perspectives, in a resource-rich and a resource-poor hospital.
\end{abstract}

\title{
Methods
}

Qualitative interviews and focus groups were conducted with patients and their treating physicians from two urban hospitals. Patients were identified through retrospective review of pathology and tumor registries; their charts abstracted to ascertain treatments. Treating physicians were identified and contacted to discuss the treatment decision process. Physicians were also asked to discuss patients who did not receive definitive treatment. Transcripts were analyzed deductively using themes from the Health Belief Model, and inductively to explore emergent themes.

\section{Results}

Overall, patients and physicians discussed similar factors that influenced the decision making process at both hospitals. However, a few important differences were found: providers at the resource-poor hospital discussed cost as a barrier, highlighted having limited treatment options for their patients, and noted issues with follow-up as external 
factors affecting treatment decisions. Patients at the resource-poor hospital expressed greater fear and anxiety, and less self-efficacy and motivation in comparison to patients treated at the hospital with greater resources. Importantly, patients at both hospitals described significant trust in their physician, yet only at the resource-poor hospital did patients suggest that they lacked knowledge regarding treatment side-effects, despite physicians at both hospitals describing their attempt to disclose all side-effects.

\section{Conclusion}

These findings identify both medical-system factors, and practice-level factors that can help guide the development of interventions to reduce prostate cancer treatment disparities.

\section{Keywords:}

Prostate cancer

Decision-making

Disparities

Underserved populations 


\section{INTRODUCTION}

Prostate cancer is the most common cancer among men in the United States, and over 25,000 deaths are estimated in 2015 due to the disease [1]. This burden falls disproportionately on black men, who have greater than twice the risk of developing and dying from prostate cancer as all other races or ethnicities [1]. Moreover, black men tend to be diagnosed with prostate cancer at an earlier age, present with more advanced stages of disease, and have poorer treatment outcomes as compared with Caucasian men $[2,3]$. Socio-economic and cultural reasons, including access to and perceptions about screening, distrust of the healthcare system, and lack of knowledge about prostate cancer itself, contribute to the etiology of this disparity [4-7].

One factor within the medical care arena known to contribute to this disparity is differences in treatment of blacks with prostate cancer [8]. Uncertainty exists around the best treatment option for locally advanced prostate cancer, and patients often defer to their physician's recommendation, or seek advice from external sources such as their spouse or the Internet [9-12]. Physicians typically aim to educate and engage the patient in decision-making about their care $[5,13]$, but this process can be difficult. As a result of this complexity and uncertainty, several decision aids have been developed to help both clinicians communicate about and patients understand treatment choices [5, 14]. Yet despite the availability of these tools, black men continue to make different treatment choices than their Caucasian counterparts [2, 15-18].

This study aims to explore the treatment decision-making (TDM) process from the perspectives of both patients and their physicians, and compare this process at a resource-rich versus a resource-poor hospital. Previous literature has evaluated the 
TDM process for men in general $[9,19]$, but little is known about how black men specifically, in conjunction with their physician, approach their treatment decisions [15, 20]. Moreover, environmental factors such as payer mix, availability of treatment options, or geographic variation in patient and physician preferences, are often correlated with race but may affect the TDM process independent of race [21]. Our study targeted men with intermediate- and high-risk, locally advanced prostate cancer (Gleason scores $\geq 7$ ) who could benefit from active treatment. Through qualitative interviews with both physicians and patients, we sought to obtain their perspectives on facilitators and barriers to receiving treatment, and explore the attitudes that drive treatment recommendations and selection. The results of this study can inform interventions aimed to decrease treatment disparities.

\section{METHODS}

\section{Study Design:}

This study used qualitative, semi-structured interviews to explore locally advanced prostate cancer treatment decision-making processes. Two groups of study participants were interviewed: patients who received definitive cancer therapy, and their treating physicians who also treated men who went untreated. We sought to speak with untreated men but were unable to reach any despite at least 10 calls on varying days of the week and times of day (i.e., morning, afternoon, evening, night).

Patients were recruited from two sites selected for their close geographic proximity, yet divergent patient populations: an academic medical center (site 1) and a municipal hospital (site 2); both operate in the same broad metropolitan area, yet serve 
different demographic populations. Site 1 is a not-for-profit hospital with over 1000 beds, treating a predominantly white population, with roughly one third of patients insured by Medicaid [22]. Site 2 is a safety-net hospital with under 300 beds treating a predominantly black population with roughly two-thirds of patients insured by Medicaid [22].

At the academic center, we examined pathology records from 2007-2012 to identify men who had prostate biopsies with a definitive tissue cancer diagnosis and a Gleason score in the range of 7-10. We identified 345 black men and a random sample of Gleason matched white men within 10 year groups $(<60,60-69,70-79,80+)$ were selected $(\mathrm{N}=339)$. At the municipal hospital, we identified all 104 black men with Gleason score of 7-10 from the Tumor Registry from 2007-13. Charts were abstracted; men with metastatic disease and those with poor prognosis due to other illnesses were excluded leaving a final sample of 575 (293 black and 282 white) men from the academic center and 66 black men from the municipal hospital. Patients with a definitive treatment were contacted.

The first phase of the study involved identification of men who received definitive treatment. Definitive treatment was defined as one of the following treatment methods: any form of radical prostatectomy, radiation external beam radiation therapy (EBRT) or brachytherapy with androgen deprivation therapy; or monotherapy with androgen deprivation therapy if prior to 2009 (changes in the field merited the removal of this therapy in 2009, see [23]).

\section{Participant Recruitment}


Focus group guides included six topics, previously shown to affect the decisionmaking process in other populations[13, 24, 25]: understanding the success of different treatment; expectations of side effects with different treatments; how the treating urologist is determined; attitudes towards living with uncertainty (e.g., active surveillance vs. active treatment); attitudes about the treatment received; and trust of their physician.

Because we were interested in ascertaining black men's barriers to care and treatment decision-making, we identified and preferentially recruited men who had longer times between diagnosis and treatment as these individuals may have wrestled more with their decision-making.

We contacted each focus group participant's physician(s) for his prostate cancer (either medical oncology, urology, and/or radiation oncology) to interview. Physician interviews were conducted from August through December 2014. The physician interview guide was designed to assess the physician's general approach to counsel patients about treatment options, the factors affecting their treatment recommendation, reasons a treatment may be recommended over another, what they believe influences the decisions their patients make, and how referrals are made. Providers were also asked to review patient cases in which the patient did not receive treatment. All focus groups and physician interviews were audio recorded, transcribed verbatim, and coded using Atlas.ti software. Informed consent was obtained for all interviewees and focus group participants.

\section{Data Analysis}


Analysis of both the focus groups and interviews proceeded using both deductive and inductive approaches. Deductively, an initial code list was created based on a review of the literature around treatment decision-making, and followed the Health Belief Model (HBM) [26]. The HBM provides an established framework that can be useful in understanding factors associated with individual health behavior, such as decision making. This model has also previously been applied to understanding prostate cancer TDM [20, 27]. Coding of the interview transcripts was also inductive, allowing the development of new codes based on patterns that emerged from the data. Throughout the coding and analysis process, the coding dictionary was refined as emergent codes were added. Each focus group and interview transcript was coded by two experienced researchers to ensure consistency in coding, and agreement about coding decisions and emergent themes.

\section{RESULTS}

\section{Population Studied}

Overall, 298 black men were identified from pathology and the tumor registry. Of these men, 29 men did not receive definitive treatment as defined above for this study. The remaining 267 men were eligible for focus group participation, and 12 were recruited to participate in either focus groups or one-on-one interviews (six from site 1; six from site 2). The demographic and treatment characteristics of these patients are shown in Table 1. Originally, we intended to conduct about eight patient focus groups, at least four with black patients, and four with white patients. We aimed to include minority men both without active treatment as well as those with active treatment. We planned to 
recruit men who experienced "poorer" quality of care. The low numbers with underuse made it impossible to reach the numbers we had anticipated. We tried numerous times to contact the 25 underuse patients - unsuccessfully. Most had moved and were unreachable or died.

We conducted semi-structured interviews with 15 of the 17 physicians who treated 22 of the 25 underuse patients: 10 urologists, two medical oncologists, and three radiation oncologists with an average of $20.5 \mathrm{yrs}$ in practice. Of the interviewed physicians, 13 treated patients at site 1 , and two treated patients at site 2 .

\section{Treatment Decision Making in the Context of the Health Belief Model}

Using the domains of the HBM to frame the data, we found similarities and differences across sites in both provider (Table 2) and patient (Table 3) interviews. Below we discuss this site comparison in greater detail, examining each of the four factors of this model in turn: external, social, knowledge, and clinical.

External Factors: For patients at the two hospitals, differences emerged in the discussion of external factors that influenced TDM, particularly around the issues of peer cues to action, and financial considerations. Patients at the resource-rich hospital discussed the impact of seeing family members, friends, and even celebrities, going through treatment (or lack of treatment), and how that experience motivated their treatment decisions.

When I found out I had prostate cancer, my first thing was to call somebody who I knew who had prostate cancer... talked to dozens of guys, probably more than twenty, people sometimes telephone, email, people as far away as China that my wife had found, and two of them mentioned Dr. [Name]. They were older than me, but they had minimal side effects, and that's what led me to choosing the treatment here, including the former mayor of New York City, whose name I won't mention, who had the exact same treatment that I did. I met with him and he 
said, "This is what happened to me". And he said, "These may or may not be your side effects, but I would stick with this guy.

Financial considerations were mentioned primarily as a barrier for patients at the resource-poor hospital. In contrast, at the resource-rich hospital, patients described having options and seeking out medical opinions from several doctors located in geographically distant locations. This availability of options, and traveling to seek other opinions was not mentioned among patients from the resource-poor hospital.

Physicians at both hospitals recognized the important role that peers can play in motivating treatment decisions, and also discussed how spouses exert a strong influence on treatment decisions. Providers at the resource-rich hospital did mention cost as an issue but they described how systems were in place to prevent cost from being prohibitive to their patients. Providers at the resource-poor hospital, similar to their patients, noted cost as a barrier to treatment. These providers also noted that certain treatment options were not available at their hospital, limiting patient treatment options. In addition, they described the impact of patients' life circumstances on their treatment recommendations. One urologist noted that he didn't offer surgery to a homeless patient as there was no way the patient could be in a homeless shelter with a Foley catheter draining urine into a bag.

In addition, providers at the resource-poor hospital described challenges related to patient follow-up, particularly for patients who did not receive treatment. These physicians described the systems in place to ensure patient follow-up, yet felt limited in their ability to reach patients that did not respond to their outreach efforts. Those physicians also voiced frustration about their inability to determine if a patient is lost to follow-up, or received care from a different provider. This latter concern is particularly 
salient given that most physicians recommend that patients seek other professional opinions as part of the TDM process.

That's an age old question. How do you keep people in the system? Because when someone's lost, you don't know if they're truly lost and not receiving any care, or they switched elsewhere. Because the way I look at it is someone didn't like me for whatever reason or didn't like the institution, that's okay just as long as they're getting cared for... but I have no way of knowing that. I don't know of a good system here.

Social Factors: Social factors discussed by the patients at the two hospitals appeared more individually dependent rather than institutionally based, as similar themes were described by patients at both sites. Patients at both hospitals described being motivated to seek treatment, often in order to avoid the experiences they had observed of relatives or friends that had prostate (or other) cancers. Patients at both hospitals discussed their information-seeking process, including their desire to have control over their treatment decisions:

I spent quite a bit of time, I spent hours on the computer. Looking from testimonies, from cancer treatment...to the oncologist, to the things from [Hospital Website], I looked at sources, you know, I read a whole lot about it, what other treatments are there. So based on other outside sources, I still haven't done anything. I want to make sure that when I do something, that I am comfortable with what I do. I don't want anyone to force me into it.

Alternatively, some patients at both hospitals were more passive in their information seeking approach, based on their view of the outcomes:

I really thought it was half a dozen one way or the other because I had a friend... my best friend had radiation and he was describing what happened with him, and I said "You know what, it sounds like there's about a wash. So, you know, either way, it's going to be a change, so l'll go with the surgery and just have a clear pathology report. 
Despite these differences in perceptions regarding control and their decision styles, patients from the resource-poor hospital also expressed fear and anxiety regarding treatment decisions, and these emotions were not noted by patients at the resource-rich hospital:

INTERVIEWEE1: I was too scared. Listen, maybe if you—looking at it clinically I should have. But looking at it from a point of fear-

INTERVIEWEE2: Yeah, I got scared! They set fear in your heart when they tell you it's cancer. Once you say cancer, anybody's going to say well can you fix it? Help me, help me. You know, not knowing the consequences that are happening to them.

Despite the differences observed between patients from the two hospitals, physician interviewees from both sites revealed general agreement about the social factors influencing TDM. Physicians recognized the variability in the treatment decision styles of patients: most patients had some prior knowledge, albeit with some misconceptions regarding the disease and treatments, and some patients would make treatment decisions regardless of physician recommendation due to other personal factors, such as the desire to be in control. However, physicians would recommend that all patients seek other opinions.

Interestingly, in alignment with patient comments from the resource-poor hospital about how fear and anxiety impacted their TDM, physicians across sites discussed how fear could affect their patients. Several physicians noted that fear can make patients choose more aggressive and unrecommended treatments. Physicians also noted that fear can make some patients retreat from medical care, particularly if those patients exhibit low self-efficacy and feel powerless to do anything about their cancer diagnosis. 
Knowledge Factors: Patients at both sites noted the importance of their relationship with their physician in the TDM process. At both sites, patients voiced significant trust and confidence in their physician, and felt that the physicians did a thorough job explaining the risks, benefits, and side-effects of each treatment. Patients felt that from both their physician, and through their own information seeking, they had sufficient information to make an informed decision regarding treatment modality. Overall, they felt that their choice of treatment depended more on their perceptions of side-effects, rather than the treatment itself because they felt that all treatments were equally beneficial.

Despite emphasizing trust, patient views at both hospitals were also inconsistent: at the resource-rich hospital they also expressed distrust in their physician and in the medical system in general:

INTERVIEWEE 5: If I do - if I had to do anything in America, I would get a piece of paper and a lawyer and have the doctor that l'm talking to sign the paper that he is doing the operation... because once you're out, you don't know who's working on you, and that's America...And that would be my only concern - is the person I'm talking to the one who does the work? Otherwise trusting - I would trust him.

INTERVIEWEE 3: Come to think about it, I never thought about that. When you're out you don't know who's working on you.

At the resource-poor hospital, in contrast, patients did not express distrust, but did comment that they believed they had not been told the complete story about treatment options. Several patients discussed that they did not know about the two primary sideeffects (impotence and incontinence), and had they known about these they would have not have elected to receive treatment, likely resulting in death. As one patient noted:

My little two cents says here what I think that what they did was good for me, but they didn't tell me the whole deal. But I can see why they didn't tell me, to keep me from making the stupid mistake. They saved my life in spite of myself. 
Because trust me, if you would've told me hey man see that gun, you won't be getting home. You see, the stupid man in me would've taken over, he would've said, hold up you know what you're getting ready to do? In the thinking of do you know what you're getting ready to do? Just that single question within myself would've put indecision and I would've been dead.

Provider views regarding knowledge factors aligned across the two sites. All providers recognized the importance of their relationship with the patient, and they also felt that incorporating the spouse into the consultation helped to build trust. Providers expressed their opinion that their primary role is to consult, not decide for a patient. To achieve this goal they would walk patients through all the risks, benefits, and sideeffects of different treatments. Providers felt that it was better to provide patients with all information prior to treatment to avoid conflicts. However, providers overall felt that patient spouses and peer experiences had a much greater influence on treatment selection than information they provided as clinicians. Providers at both sites discussed the use of decision aids, including handout material and peer education groups, to inform patients. Based on their experiences, however, they reported feeling that patients did not value these materials or the groups, and so in many cases, physicians stopped printing handouts or referring patients to peer education groups.

Clinical Factors: For patients at both sites, the primary clinical factor that played into their TDM was their likelihood of experiencing side-effects, particularly impotence and incontinence. As patients discussed clinical factors, a differentiating factor that emerged was an apparent variation across the two sites in health literacy: while patients at the resource-rich hospital described their disease state in very technical language, those at the resource-poor hospital struggled with the meaning of key terms (e.g., impotence and incontinence). 
Not surprisingly, physicians at both sites discussed clinical factors in greater depth than patients. Severe comorbidities from chronic diseases, other cancers, or previous cancer treatments played a large factor contributing to physician recommendations. Physicians from the resource-poor hospital were cognizant that their patients had higher rates of comorbidities, and more severe risk profiles, including age, smoking status, and family history:

Keep in mind that our population have high levels of diabetes and hypertension and they smoke or they have other risk factors for ED [erectile dysfunction] as well and so, you know, letting them know that something that treats their problem can actually create, this issue could be worse makes them a little concerned.

\section{DISCUSSION}

This study explores, from both patient and physician perspectives, the decision making process for black men at a resource-poor hospital who received treatment for locally advanced prostate cancer, and compared that process to a similar group of men at a resource-rich hospital. This design allowed for the identification of factors that contribute to TDM that may be related to the environment. Using the Health Belief Model, we found important similarities and differences in the factors that contribute to TDM across the two hospitals.

Perhaps what is most striking are the similarities shared by patients at the two sites. Both hospitals had patients who were motivated to search out information and reached out to find and talk with men with prostate cancer. Similarly, both had patients who were more passive and relied on the information their physicians supplied. Both were significantly concerned with side effects. Both groups were concerned about the diagnosis and treatment side effects but the men who were more health literate at both 
sites and had greater self-efficacy, sought out data to inform their decision-making. Those who expressed greater fear and anxiety were more limited in their search for information to inform decision-making.

Spousal input was important to both groups. The finding that spouses also play an important role in determining treatment decisions may suggest that prostate cancer education efforts should not be targeted only at men, but at women as well [28]. Women may have more frequent interactions with healthcare services in general, including preventive care [29], providing opportunities to engage them as educators, and this approach has been successful in improving prostate cancer screening efforts in Black men [28]. These education-oriented efforts could also help alleviate some patient fear and anxiety over prostate cancer, and improve their self-efficacy regarding the disease. Interestingly, physician efforts to provide handouts or create peer education groups were not viewed as useful at either hospital. In light of this finding, targeting patients more informally through peer or spousal channels may prove more advantageous in reducing disparities than formal approaches.

Both trusted their physicians but at the resource rich hospital, there was more mistrust of a system that was motivated by money. Interestingly, cost functioned as both a barrier and a motivator - depending on the hospital. At the resource-poor site, treatment costs were also presented as a barrier to care. However, at the resource-rich site, higher costs motivated a patient to seek care at sites with government controlled costs. At both sites, physicians said they explained risks and benefits of the various treatments. At the resource rich site, these discussions registered with the men. However, at the resource poor site, despite the patients saying that in retrospect their 
physicians told them they had discussed the side effects, several stated they were unaware of and unprepared for the unwanted side effects they experienced. Regardless of their sense of unpreparedness, these men were satisfied with their physicians, having recast their lack of information as their doctors' ways of ensuring they would proceed with needed treatments.

As with the patients, the physicians' perceptions of factors affecting patient decision-making at both hospitals were strikingly similar. However, the greatest differences were the limitations posed by external factors noted by those practicing at the resource poor facility. Physicians at the resource-poor hospital felt limited in their ability to track patients across the medical care landscape to determine if a patient was receiving care elsewhere or if the patient was lost to follow-up. Adoption of electronic health records, particularly those that can connect across organizational boundaries through health information exchanges, may improve the ability to track patients [30]. However, this technology may be limited in its ability to detect patients who remain outside of the healthcare system - a particularly relevant issue in an environment with historically high rates of uninsured individuals. A connected healthcare system could potentially increase the number and diversity of points of contact between patients and providers, and may thus be able to help reduce instances of loss to follow-up.

The observation from the resource-rich hospital that the public experience of the 'mayor' could influence treatment decisions, and facilitate dialogue about the disease suggests that a similar approach could be used in designing health promotion campaigns around this issue (Wray et al., 2009; Wray, Vijaykumar, Jupka, Zellin, \& Shahid, 2011). Men may be hesitant to discuss their treatment experiences due to its 
sensitive nature, yet providers may be in a unique position to provide support and advice for patients in how to consult other patients through community-based programs [31]. For example, interventions, such as those based out of churches or barber shops, that are accessible to the black community may work to educate and encourage public discussion of treatment options [32, 33].

The results revealed that physicians had a much more robust view of the medical system and its associated issues than patients did, most likely because of their own involvement in that system. However, patients and physicians at both sites did recognize how availability of certain treatments in an accessible (both geographically and financially) location did influence their treatment options. Physicians, on the other hand, were much more critical of the systematic factors affecting treatment accessibility.

Beyond the external factors affecting TDM, several of the social and knowledge factors also could be targeted directly to develop interventions aimed to reduce treatment disparities. Both patients and physicians articulated how personal decision styles, as well as fear and anxiety, played important roles in decision making. Instinctively, education could help to reduce fear as well as empower patients across the spectrum of decision styles. However, patients' views from the resource-poor hospital on knowledge regarding the disease, and treatment risk, benefits, and sideeffects were inconsistent, as they felt that knowledge would have led to a deleterious and regrettable treatment decision, e.g., no treatment. Additionally, even patients from that site that felt that they were well informed by their physician stated that they were glad they did not have complete knowledge of the side-effects of treatment. These inconsistencies make designing interventions difficult and complex, as there are likely 
fear issues, health literacy issues, and socio-cultural issues at play. Physicians at both sites did recognize that their role was to inform, rather then decide for patients, despite the fact that some patients simply preferred deference to their physician's recommendation. This attitude by providers helps to ensure that patients are not coerced into receiving treatment. Interventions aimed at using peers as educators and making discussion of prostate cancer less taboo could empower patients to digest information about the disease and use it to inform a decision, rather than to reject the knowledge.

\section{Limitations:}

This study faces two primary limitations rooted in the geographic location of this study and the state of the science regarding cancer care disparities. First, this study took place in two hospitals serving a large, urban area, and the findings extrapolated from that environment may not be generalizable to other areas. Other urban areas, and other hospitals in this same urban area, may have many similarities with the experiences reported in this study, yet treatment decisions are often context-dependent and socially informed. Our results and discussion should be interpreted with this limitation in mind.

Second, this study included a small sample of patient study participants. Recruiting treated patients proved difficult for several reasons, including issues with identification of patients, accuracy of contact information, and scheduling of focus groups at convenient times and locations. Despite this issue, many ideas reached saturation, even in the small sample. The findings from this study serve as useful 
formative work for the design of interventions and for future research aimed at surveying a more representative sample regarding the issues found in this study.

Third, the retrospective approach used to identify patients that received definitive treatment biases the patient sample. The views of patients that either actively chose or passively failed to receive treatment are not represented in our analysis, largely due to the high mortality rate in this group. The reasons why these patients did not receive care were speculated by physician review and recall of specific cases, and the results should be interpreted with this limitation in mind.

Finally, several unanswered questions as to the best treatment for prostate cancer remain. This issue arises from limited evidence of head-to-head comparisons for surgery and radiation therapy among men of similar clinical risk. Despite the quagmire, this study distilled measures from medium- to high-grade randomized clinical trial evidence to begin the process of evaluating the quality of prostate cancer care among these men at greatest risk for poor outcomes.

\section{Conclusion:}

The disparity regarding the effect of prostate cancer on black versus white men remains significant. Overall, this study adds to the qualitative evidence regarding prostate cancer disparities by providing an in-depth assessment of the treatment decision making processes from the perspectives of both patients and physicians, at two hospitals geographically close yet very different in terms of their environments. The findings reported in this study show significant alignment between patients and physicians on TDM factors at both sites; however, the use of the HBM helps to identify 
particular differences in the decision process. Specifically, patients and physicians highlight the importance of peer experiences and spousal support, and the critical role of treatment availability suggesting these as useful avenues around which to develop interventions. 


\section{References}

1. American Cancer Society, Cancer Facts \& Figures 2015. 2015, Atlanta: American Cancer Society.

2. Mahal, B.A., et al., Trends in disparate treatment of African American men with localized prostate cancer across National Comprehensive Cancer Network risk groups. Urology, 2014. 84(2): p. 386-92.

3. Siegel, R., D. Naishadham, and A. Jemal, Cancer statistics, 2013. CA Cancer J Clin, 2013. 63(1): p. 11-30.

4. Richardson, J.T., J.D. Webster, and N.J. Fields, Uncovering myths and transforming realities among low-SES African-American men: implications for reducing prostate cancer disparities. J Natl Med Assoc, 2004. 96(10): p. 1295-302.

5. Rim, S.H., et al., Considering racial and ethnic preferences in communication and interactions among the patient, family member, and physician following diagnosis of localized prostate cancer: study of a US population. Int J Gen Med, 2011. 4: p. 481-6.

6. Shavers, V.L. and M.L. Brown, Racial and ethnic disparities in the receipt of cancer treatment. J Natl Cancer Inst, 2002. 94(5): p. 334-57.

7. Berry, D.L., et al., Treatment decision-making by men with localized prostate cancer: the influence of personal factors. Urol Oncol, 2003. 21(2): p. 93-100. 
8. Chornokur, G., et al., Disparities at presentation, diagnosis, treatment, and survival in African American men, affected by prostate cancer. Prostate, 2011. 71(9): p. 985-97.

9. Cohen, $\mathrm{H}$. and N. Britten, Who decides about prostate cancer treatment? A qualitative study. Fam Pract, 2003. 20(6): p. 724-9.

10. Friedman, D.B., et al., Developing partnerships and recruiting dyads for a prostate cancer informed decision making program: lessons learned from a community-academic-clinical team. J Cancer Educ, 2012. 27(2): p. 243-9.

11. Friedman, D.B., et al., It takes two to talk about prostate cancer: a qualitative assessment of African American men's and women's cancer communication practices and recommendations. Am J Mens Health, 2012. 6(6): p. 472-84.

12. Steginga, S.K., et al., Making decisions about treatment for localized prostate cancer. BJU Int, 2002. 89(3): p. 255-60.

13. Hall, J.D., et al., Why patients choose prostatectomy or brachytherapy for localized prostate cancer: results of a descriptive survey. Urology, 2003. 61(2): p. 402-7.

14. Zeliadt, S.B., et al., Why do men choose one treatment over another?: a review of patient decision making for localized prostate cancer. Cancer, 2006. 106(9): p. 1865-74.

15. Moses, K.A., et al., Impact of ethnicity on primary treatment choice and mortality in men with prostate cancer: data from CaPSURE. J Clin Oncol, 2010. 28(6): p. 1069-74. 
16. Presley, C.J., et al., A new approach to understanding racial disparities in prostate cancer treatment. J Geriatr Oncol, 2013. 4(1): p. 1-8.

17. Underwood, W., et al., Racial/ethnic disparities in the treatment of localized/ regional prostate cancer. J Urol, 2004. 171(4): p. 1504-7.

18. $\mathrm{Xu}, \mathrm{J}$., et al., Racial differences in treatment decision-making for men with clinically localized prostate cancer: A population based study. Journal of Racial and Ethnic Health Disparities, 2015.

19. Bowen, D.J., et al., Prostate cancer screening and informed decision-making: provider and patient perspectives. Prostate Cancer Prostatic Dis, 2011. 14(2): p. $155-61$.

20. Xu, J., et al., Men's perspectives on selecting their prostate cancer treatment. J Natl Med Assoc, 2011. 103(6): p. 468-78.

21. Cooperberg, M.R., J.M. Broering, and P.R. Carroll, Time trends and local variation in primary treatment of localized prostate cancer. J Clin Oncol, 2010. 28(7): p. 1117-23.

22. American Hospital Directory. Free Hospital Profiles. 2015 [cited 2016 1/15]; Available from: https://www.ahd.com/search.php.

23. Widmark, A., et al., Endocrine treatment, with or without radiotherapy, in locally advanced prostate cancer (SPCG-7/SFUO-3): an open randomised phase III trial. Lancet, 2009. 373(9660): p. 301-8.

24. Demark-Wahnefried, W., et al., Treatment options, selection, and satisfaction among African American and white men with prostate carcinoma in North Carolina. Cancer, 1998. 83(2): p. 320-30. 
25. Holmboe, E.S. and J. Concato, Treatment decisions for localized prostate cancer: asking men what's important. J Gen Intern Med, 2000. 15(10): p. 694-701.

26. Rosenstock, I.M., V.J. Strecher, and M.H. Becker, Social learning theory and the Health Belief Model. Health Educ Q, 1988. 15(2): p. 175-83.

27. Plowden, K.O., Using the health belief model in understanding prostate cancer in African American men. ABNF J, 1999. 10(1): p. 4-8.

28. Carter, V.L., et al., Increasing prostate cancer screening among African American men. J Health Care Poor Underserved, 2010. 21(3 Suppl): p. 91-106.

29. Vaidya, V., G. Partha, and M. Karmakar, Gender differences in utilization of preventive care services in the United States. J Womens Health (Larchmt), 2012. 21(2): p. $140-5$.

30. Brailer, D.J., Interoperability: the key to the future health care system. Health Aff (Millwood), 2005. Suppl Web Exclusives: p. W5-19-W5-21.

31. Horowitz, C.R., et al., Using community-based participatory research to reduce health disparities in East and Central Harlem. Mt Sinai J Med, 2004. 71(6): p. $368-74$.

32. Luque, J.S., et al., Feasibility Study of Engaging Barbershops for Prostate Cancer Education in Rural African-American Communities. J Cancer Educ, 2015. 30(4): p. 623-8.

33. Saunders, D.R., et al., Development of the men's prostate awareness church training: church-based workshops for African American men. Fam Community Health, 2013. 36(3): p. 224-35. 
Table 1: Patient Characteristics

\begin{tabular}{|c|c|c|}
\hline & $\begin{array}{l}\text { Resource-Poor } \\
\text { Hospital }(n=6)\end{array}$ & $\begin{array}{l}\text { Resource-Rich Hospital } \\
(n=6)\end{array}$ \\
\hline Mean Age (sd) & $68.0(6.3)$ & $64.8(7.2)$ \\
\hline \multicolumn{3}{|l|}{ Race } \\
\hline Black & $6(100 \%)$ & $4(67 \%)$ \\
\hline White & 0 & $2(33 \%)$ \\
\hline \multicolumn{3}{|l|}{ Insurance } \\
\hline $\begin{array}{c}3^{\text {rd }} \text { Party } \\
\text { Commercial }\end{array}$ & $2(33 \%)$ & $2(33 \%)$ \\
\hline Medicaid & $3(50 \%)$ & 0 \\
\hline Medicare & $1(17 \%)$ & $3(50 \%)$ \\
\hline Unknown & 0 & $1(17 \%)$ \\
\hline Comorbidity $(>0)$ & $4(67 \%)$ & 0 \\
\hline \multicolumn{3}{|l|}{ Gleason Sum } \\
\hline 7 & $2(33 \%)$ & $4(67 \%)$ \\
\hline 8 & $2(33 \%)$ & $1(17 \%)$ \\
\hline 9 & $2(33 \%)$ & $1(17 \%)$ \\
\hline
\end{tabular}


Table 2: Patient themes present at two hospitals.

\begin{tabular}{|c|c|c|}
\hline & Resource Poor Hospital & Resource Rich Hospital \\
\hline \multirow[t]{3}{*}{ External Factors } & Peer Cues to Action & Peer Cues to Action \\
\hline & $\begin{array}{l}\text { Limited Treatment } \\
\text { Options }\end{array}$ & $\begin{array}{l}\text { Available Treatment } \\
\text { Options }\end{array}$ \\
\hline & Cost as a Barrier & $\begin{array}{l}\text { Cost as a Motivator (e.g., } \\
\text { to seek treatment } \\
\text { elsewhere) }\end{array}$ \\
\hline \multirow[t]{4}{*}{ Social Factors } & Control (Decision Style) & Control (Decision Style) \\
\hline & Self-Efficacy & Self-Efficacy \\
\hline & Motivation & Motivation \\
\hline & Fear \& Anxiety & \\
\hline \multirow[t]{3}{*}{ Knowledge Factors } & $\begin{array}{l}\text { Patient-Provider } \\
\text { Relationship (Trust) }\end{array}$ & $\begin{array}{l}\text { Patient-Provider } \\
\text { Relationship (Trust) }\end{array}$ \\
\hline & $\begin{array}{l}\text { Perceived Knowledge of } \\
\text { Risks/ Benefits }\end{array}$ & $\begin{array}{l}\text { Perceived Knowledge of } \\
\text { Risks/ Benefits }\end{array}$ \\
\hline & $\begin{array}{l}\text { Lack of Knowledge as a } \\
\text { Beneficial }\end{array}$ & Lack of Trust \\
\hline Clinical Factors & Side Effects & Side Effects \\
\hline
\end{tabular}


Table 3: Provider themes present at two hospitals.

\begin{tabular}{|c|l|l|}
\hline \multirow{2}{*}{ External Factors } & Resource Poor Hospital & Resource Rich Hospital \\
\hline \multirow{5}{*}{ Social Factors } & Peer Cues to Action & Peer Cues to Action \\
\hline \multirow{5}{*}{ Spouse } & Spouse \\
\hline & Cost as a Barrier & \\
\hline & Options Not Available & \\
\hline & Follow-up Issues & \\
\hline \multirow{2}{*}{ Knowledge Factors } & Financial Motivation & Financial Motivation \\
\hline & Control (Decision Style) & Control (Decision Style) \\
\hline & Fear \& Anxiety & Fear \& Anxiety \\
\hline & Relationship (Trust) & $\begin{array}{l}\text { Patient-Provider } \\
\text { Relationship (Trust) }\end{array}$ \\
\hline & Perceived Risks/ Benefits & Perceived Risks/ Benefits \\
\hline & $\begin{array}{l}\text { Decision Aides (not } \\
\text { useful) }\end{array}$ & $\begin{array}{l}\text { Decision Aides (not } \\
\text { useful) }\end{array}$ \\
\hline \multirow{2}{*}{ Clinical Factors } & Co-morbidities & \\
\hline
\end{tabular}

